\title{
Air Control Toolbox (ACT_v1.0): a machine learning flexible surrogate model to explore mitigation scenarios in air quality forecasts.
}

\author{
Augustin Colette ${ }^{1}$, Laurence Rouï ${ }^{1}$, Frédérik Meleux ${ }^{1}$, Vincent Lemaire ${ }^{1,2}$, Blandine Raux ${ }^{1}$ \\ ${ }^{1}$ Institut National de 1'Environnement Industriel et des Risques (INERIS), Parc Alata, BP2, 60550 Verneuil-en- \\ Halatte, France \\ ${ }^{2}$ now at Amplisim, 96b Boulevard Raspail, 75006 Paris, France \\ Correspondence to: Augustin Colette (augustin.colette@ineris.fr)
}

\section{Supplementary Figures}




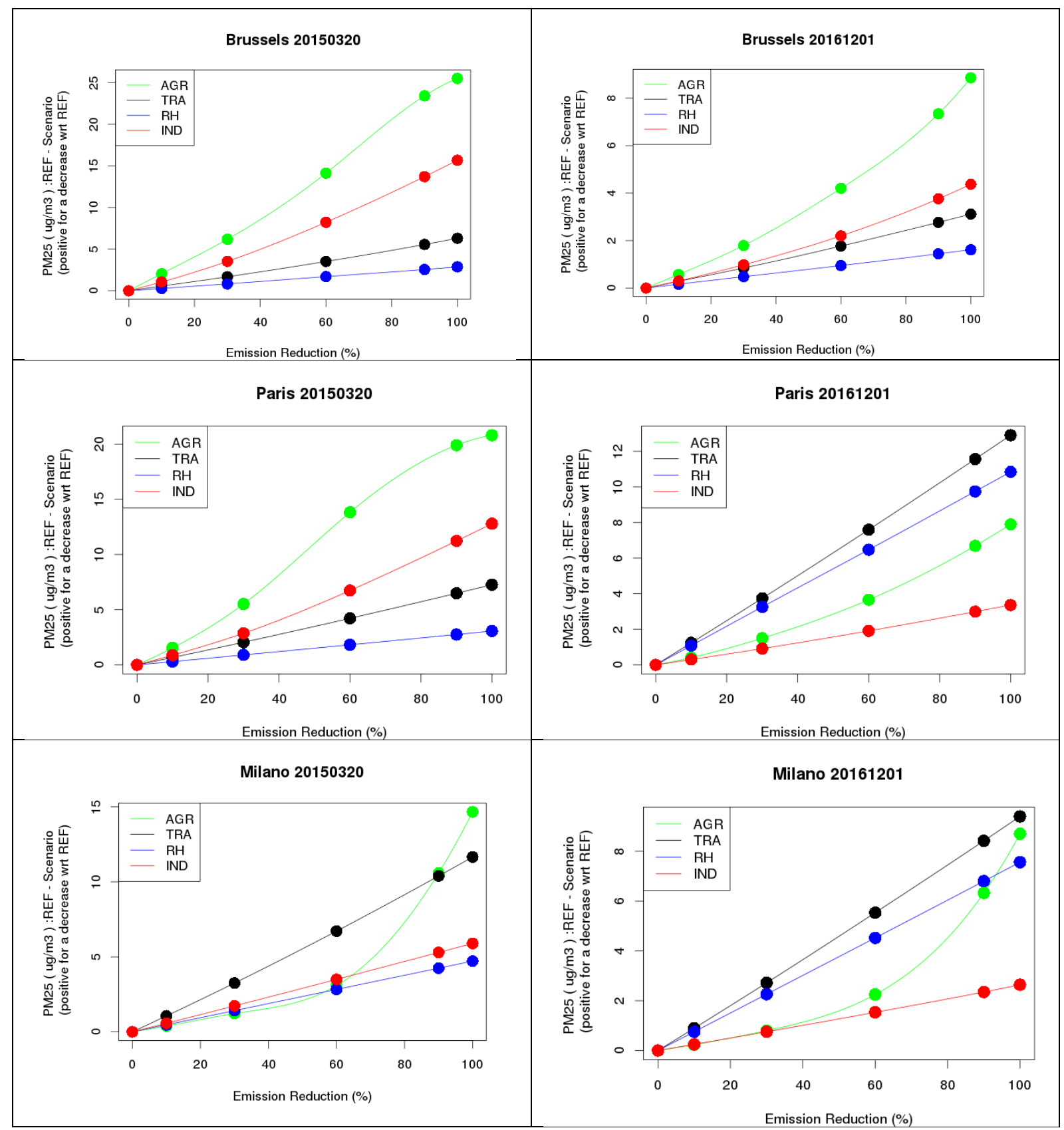

Figure S.1 : Modelled $\mathrm{PM}_{2.5}$ reduction (y-axis : positive for a decrease with respect to the reference, $\mu \mathrm{g} / \mathrm{m}^{3}$ ) for a given reduction in Agriculture (green), Industrial (red), Residential Heating (blue), and Traffic (black) emissions (x-axis: in $\%$ ) in Brussels, Paris and Milano (top to bottom) and for 20150320 (left) and 20161201 (right). 


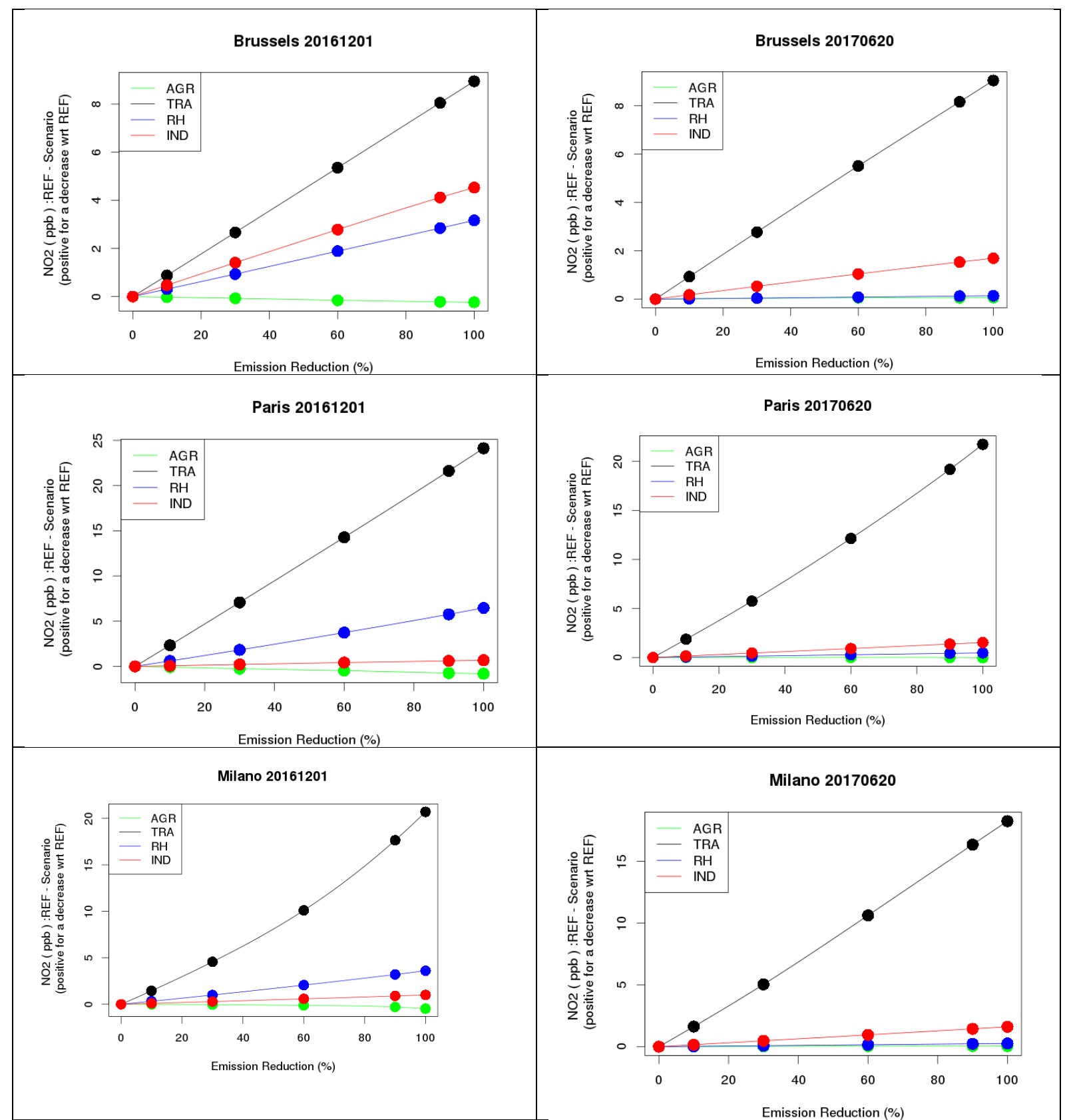

Figure S.2 : Same as Figure S.1 for $\mathrm{NO}_{2} 20161201$ and 20170620 


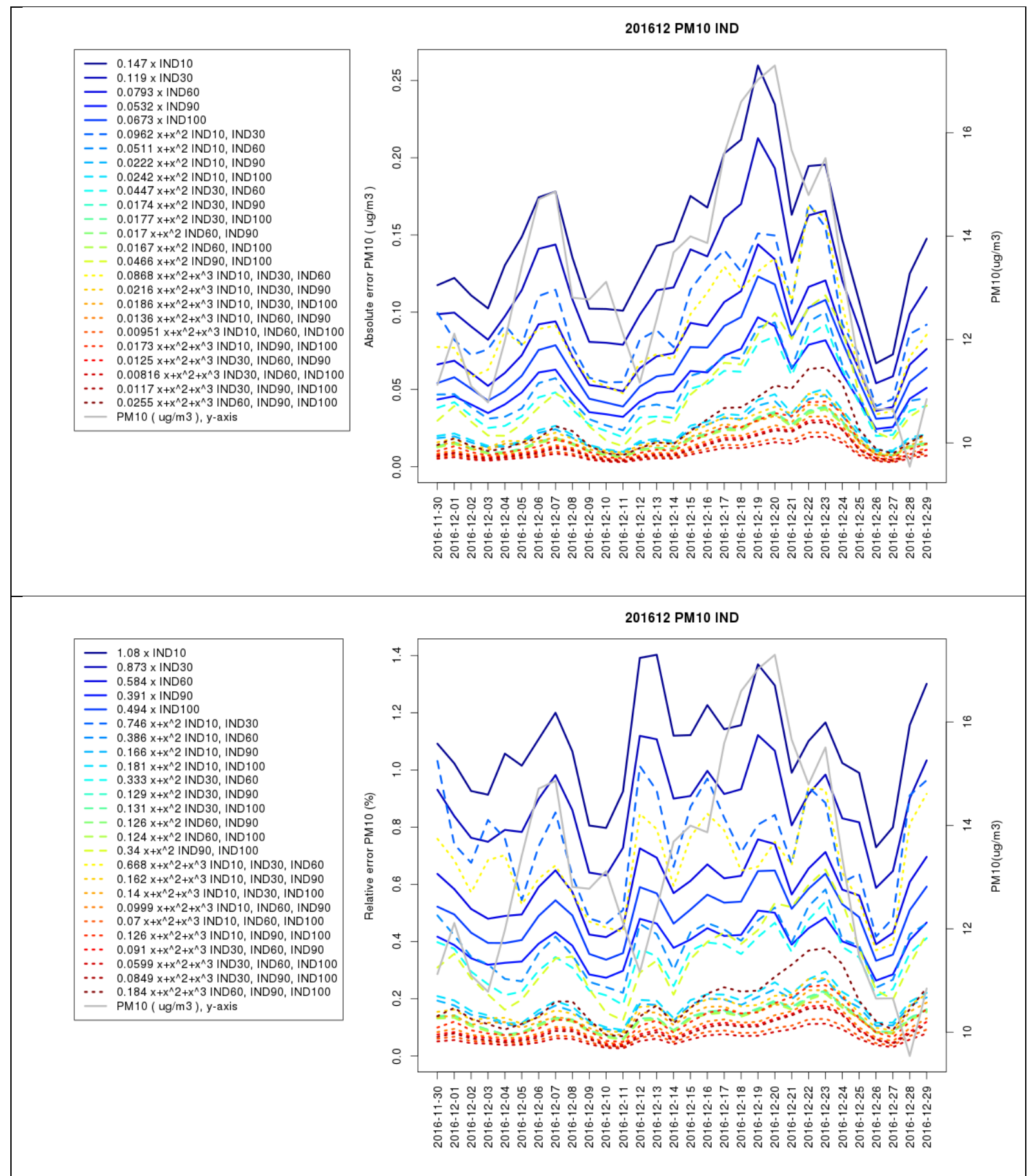

Figure S.3 : Absolute (top, $\mu \mathrm{g} / \mathrm{m}^{3}$ ) and relative (bottom, \%) error over Western Europe of the univariate surrogate model for the Industry activity sector in December 2016. The colored lines are for individual surrogate models, with the complexity and training scenario provided in the legend, as well as the error averaged over the whole time-period. The grey curve gives the day-to-day variation of $P_{10}\left(\mu \mathrm{g} / \mathrm{m}^{3}\right)$ averaged over the region (displayed on the right-hand-side $y$ axis) 


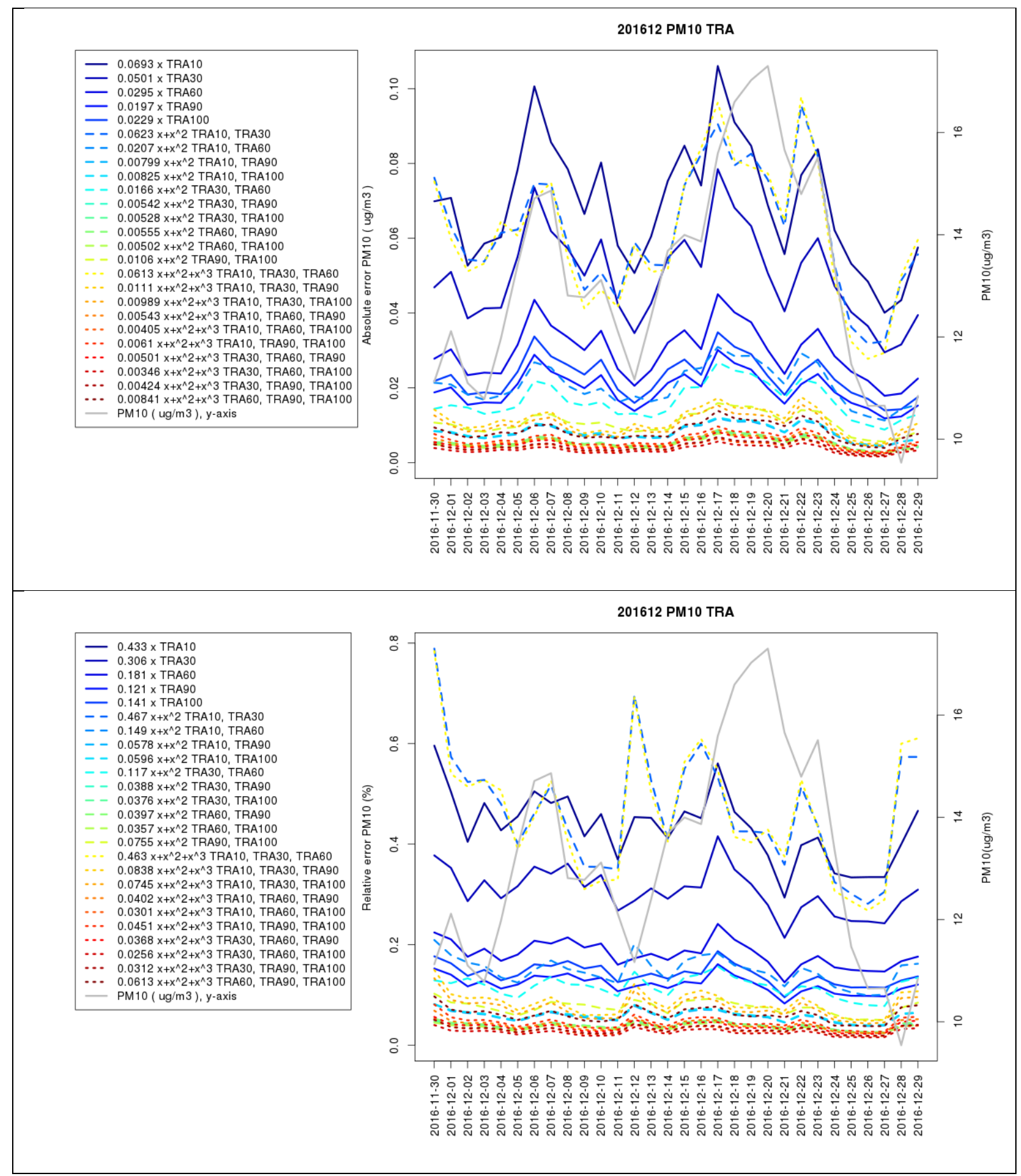

Figure S.4 : Same as Figure S.3 for Traffic 


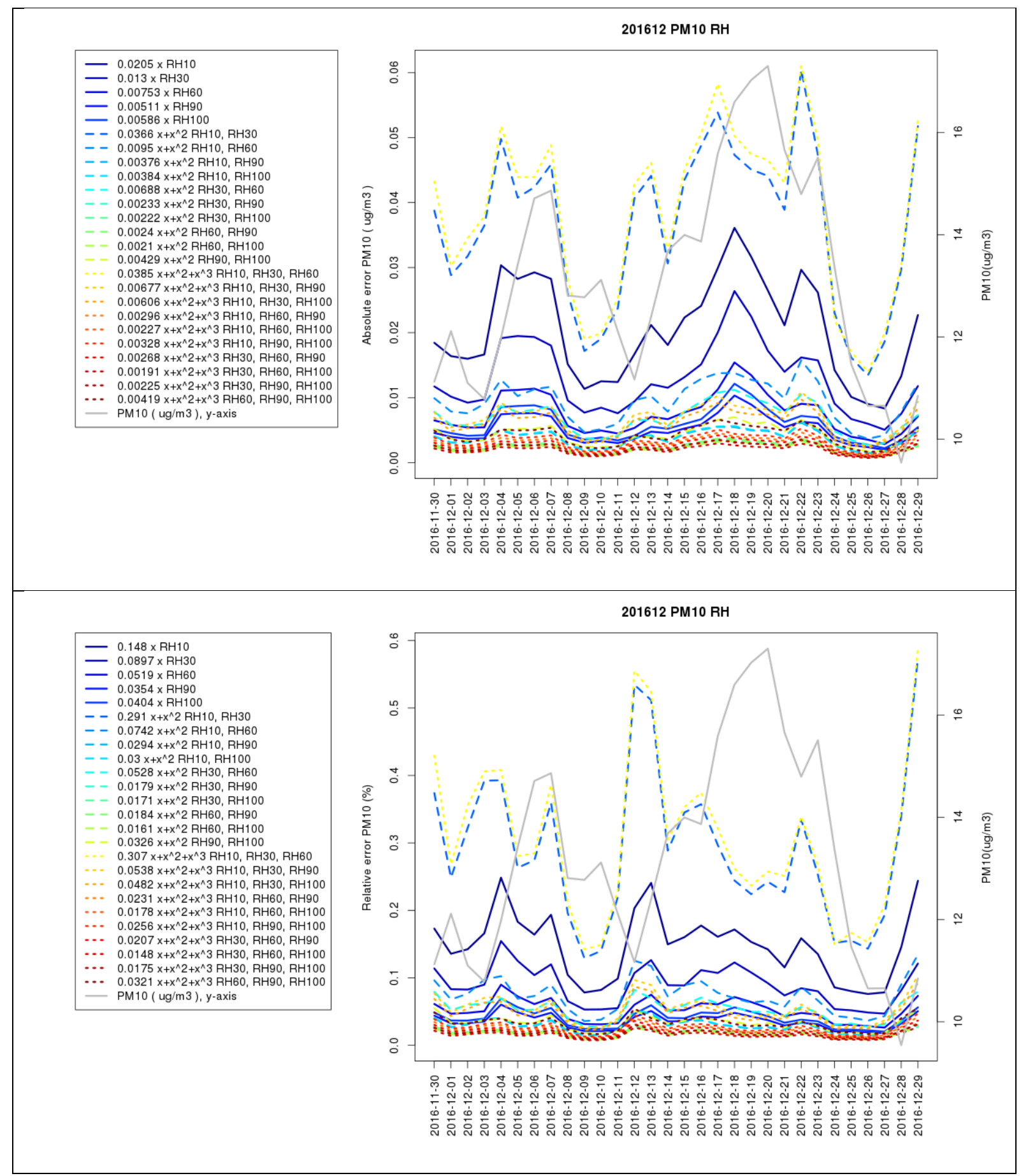

Figure S.5 : Same as Figure S.3 for Residential Heating 

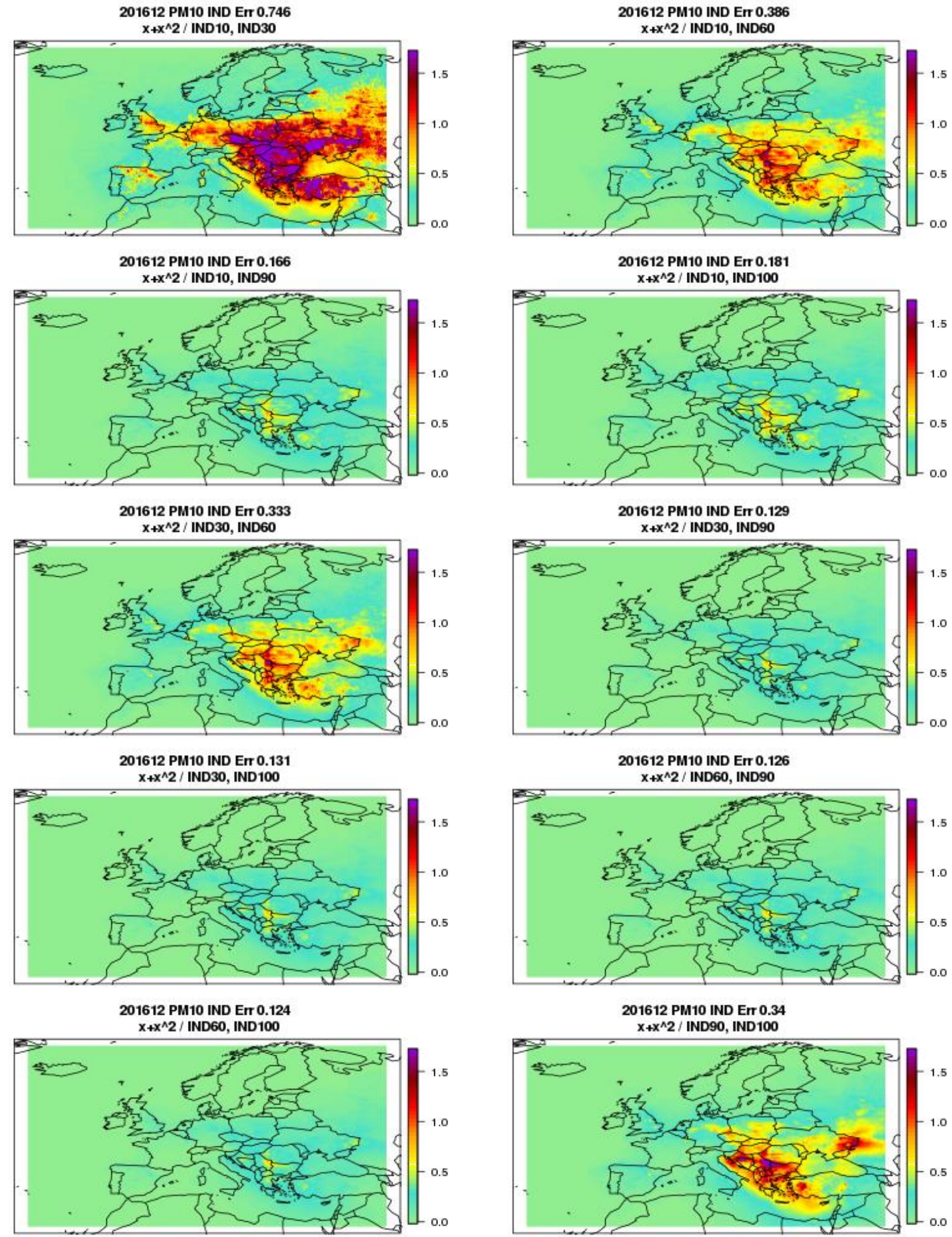

Figure S.6 : Relative error (\%) averaged over the month of December 2016 for the quadratic univariate PM10 models with respect to the industry activity sector. The sensitivity scenarios used to train the individual models are indicated in the title of each panel, as well as the average error. 

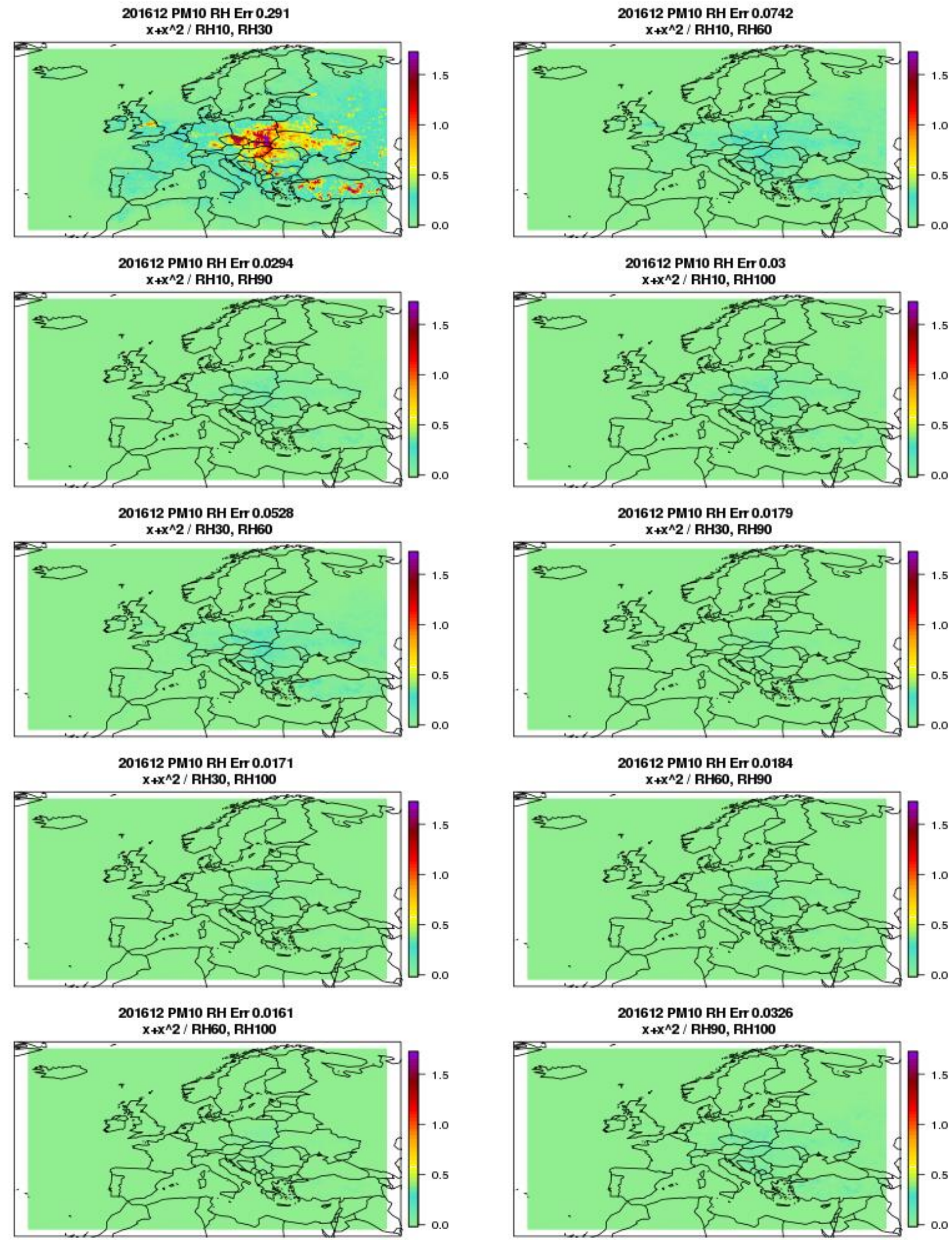

Figure S.7 : Same as Figure S.6 for Residential heating 

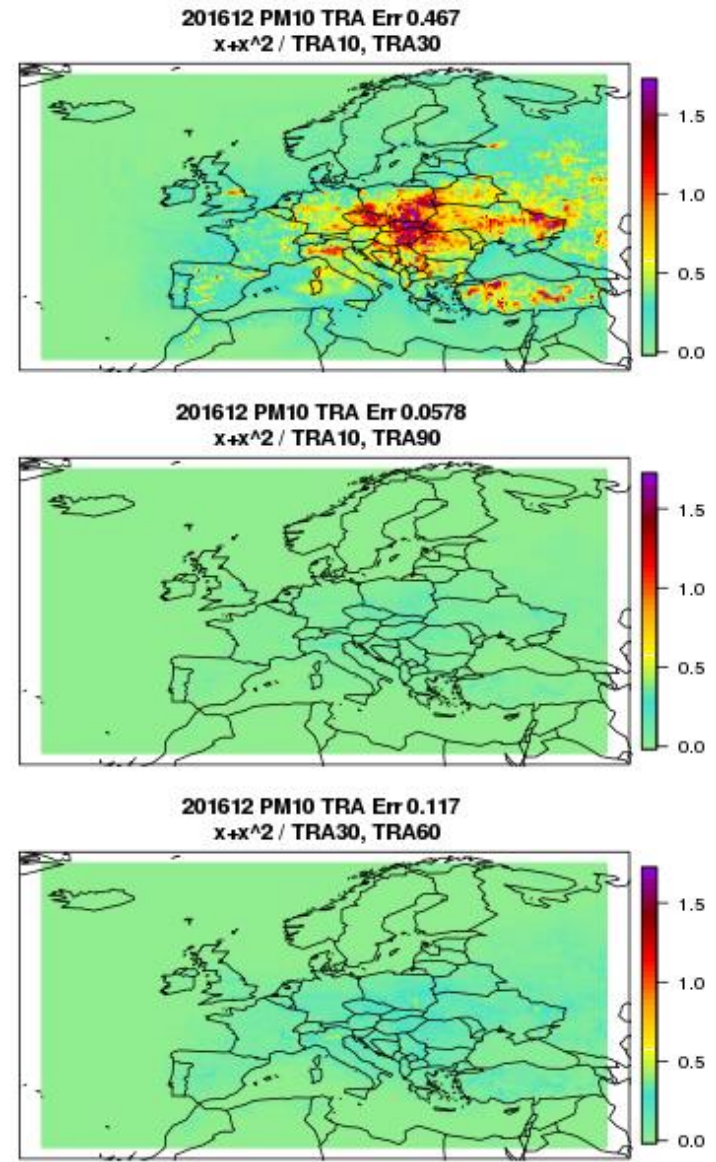

201612 PM10 TRA Err 0.0376
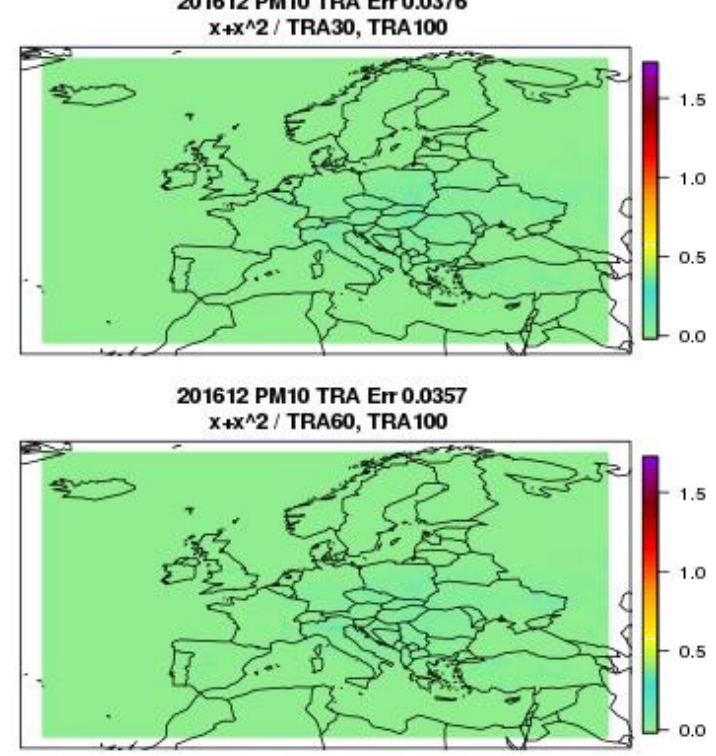
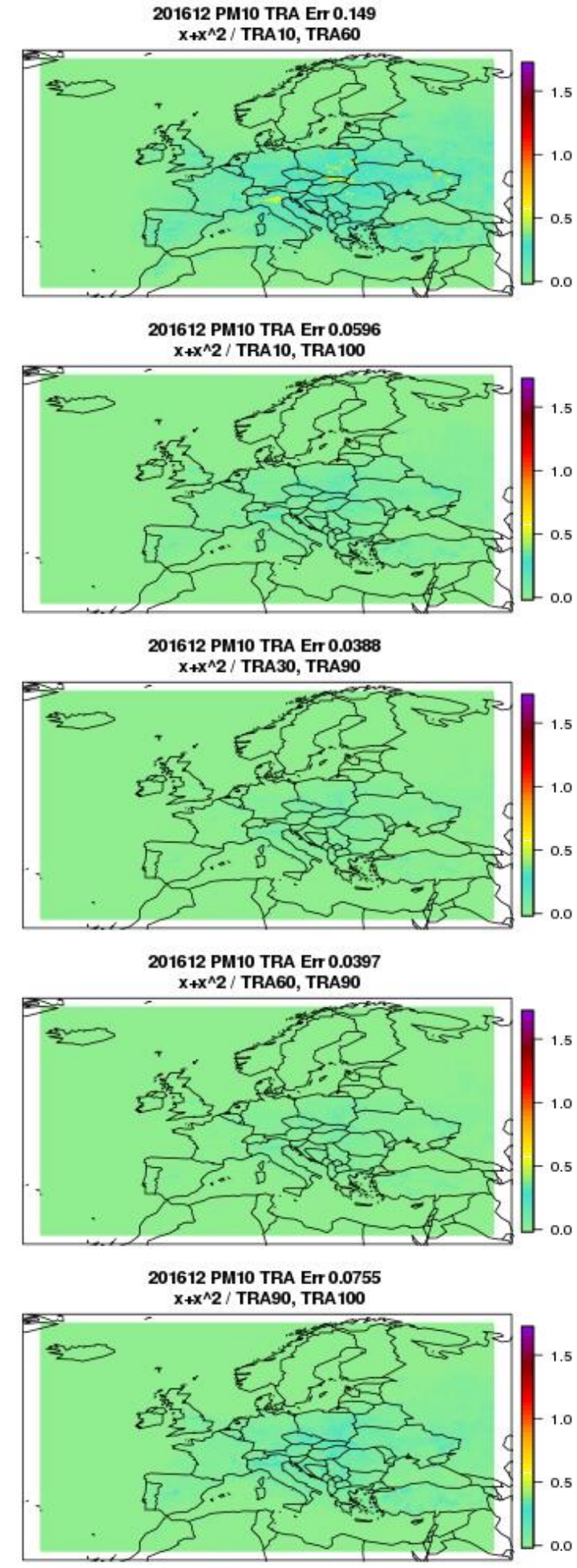

Figure S.8 : Same as Figure S.6 for Traffic 


\begin{tabular}{|c|c|c|c|c|c|}
\hline Polynomial & Training & AGR & IND & $\mathrm{RH}$ & TRA \\
\hline $\mathrm{x}$ & 10 & 3,933 & 1,133 & 0,130 & 0,339 \\
\hline $\mathrm{x}$ & 30 & 3,123 & 0,894 & 0,082 & 0,236 \\
\hline $\mathrm{x}$ & 60 & 1,983 & 0,573 & 0,047 & 0,137 \\
\hline$x$ & 90 & 1,310 & 0,382 & $\underline{0,032}$ & 0,092 \\
\hline $\mathrm{x}$ & 100 & 1,463 & 0,471 & 0,036 & 0,106 \\
\hline$x+x 2$ & 1030 & 3,540 & 0,698 & 0,284 & 0,407 \\
\hline$x+x 2$ & 1060 & 2,097 & 0,329 & 0,066 & 0,117 \\
\hline$x+x 2$ & 1090 & 0,833 & 0,137 & 0,027 & 0,046 \\
\hline$x+x 2$ & 10100 & 0,856 & 0,147 & 0,027 & 0,047 \\
\hline$x+x 2$ & 3060 & 1,917 & 0,285 & 0,055 & 0,096 \\
\hline$x+x 2$ & 3090 & 0,663 & 0,104 & 0,018 & 0,031 \\
\hline$x+x 2$ & 30100 & 0,646 & 0,105 & 0,017 & 0,030 \\
\hline$x+x 2$ & 6090 & 0,697 & 0,101 & 0,017 & 0,032 \\
\hline$x+x 2$ & 60100 & $\underline{0,634}$ & $\underline{0,099}$ & 0,015 & $\underline{0,028}$ \\
\hline$x+x 2$ & 90100 & $\overline{1,563}$ & $\overline{0,254}$ & 0,029 & 0,058 \\
\hline$x+x 2+x 3$ & 103060 & 3,437 & 0,634 & 0,315 & 0,428 \\
\hline$x+x 2+x 3$ & 103090 & 0,867 & 0,140 & 0,054 & 0,076 \\
\hline$x+x 2+x 3$ & 1030100 & 0,736 & 0,122 & 0,049 & 0,068 \\
\hline$x+x 2+x 3$ & 106090 & 0,596 & 0,081 & 0,022 & 0,034 \\
\hline$x+x 2+x 3$ & 1060100 & 0,407 & 0,057 & 0,017 & 0,026 \\
\hline$x+x 2+x 3$ & 1090100 & 0,739 & 0,099 & 0,024 & 0,038 \\
\hline$x+x 2+x 3$ & 306090 & 0,560 & 0,074 & 0,020 & 0,032 \\
\hline$x+x 2+x 3$ & 3060100 & 0,360 & 0,049 & 0,015 & 0,022 \\
\hline$x+x 2+x 3$ & 3090100 & 0,514 & 0,068 & 0,017 & 0,027 \\
\hline$x+x 2+x 3$ & 6090100 & 1,150 & 0,143 & 0,029 & 0,051 \\
\hline
\end{tabular}

Table S.1 : Relative error (\%) of the PM10 univariate polynomial surrogate models averaged over Western Europe for three air pollution episodes $(201503,201612,201701)$ for various polynomial structures, and various set of Chimere simulations used in the training. A color shading is applied to highlight the worst (red) and best (green) performances. 


\begin{tabular}{|c|c|c|c|c|c|}
\hline Polynomial & Training & AGR & IND & $\mathrm{RH}$ & TRA \\
\hline $\mathrm{x}$ & 10 & 0,083 & 0,500 & 0,002 & 0,738 \\
\hline $\mathrm{x}$ & 30 & 0,070 & 0,390 & 0,001 & 0,571 \\
\hline $\mathrm{x}$ & 60 & 0,053 & 0,241 & 0,001 & 0,347 \\
\hline $\mathrm{x}$ & 90 & 0,037 & 0,159 & 0,001 & 0,229 \\
\hline $\mathrm{x}$ & 100 & 0,045 & 0,191 & 0,001 & 0,273 \\
\hline$x+x 2$ & 1030 & 0,115 & 0,184 & 0,002 & 0,263 \\
\hline$x+x 2$ & 1060 & 0,077 & 0,104 & 0,001 & 0,141 \\
\hline$x+x 2$ & 1090 & 0,034 & 0,040 & 0,000 & 0,053 \\
\hline$x+x 2$ & 10100 & 0,036 & 0,043 & 0,000 & 0,057 \\
\hline$x+x 2$ & 3060 & 0,072 & 0,090 & 0,000 & 0,122 \\
\hline$x+x 2$ & 3090 & 0,028 & 0,030 & 0,000 & 0,039 \\
\hline$x+x 2$ & 30100 & 0,028 & 0,030 & 0,000 & 0,039 \\
\hline$x+x 2$ & 6090 & 0,029 & 0,030 & 0,000 & 0,039 \\
\hline$x+x 2$ & 60100 & 0,028 & $\underline{0,029}$ & 0,000 & $\underline{0,038}$ \\
\hline$x+x 2$ & 90100 & 0,078 & 0,068 & 0,000 & 0,083 \\
\hline$x+x 2+x 3$ & 103060 & 0,114 & 0,125 & 0,002 & 0,179 \\
\hline$x+x 2+x 3$ & 103090 & 0,035 & 0,029 & 0,000 & 0,038 \\
\hline$x+x 2+x 3$ & 1030100 & 0,029 & 0,025 & 0,000 & 0,033 \\
\hline$x+x 2+x 3$ & 106090 & 0,027 & 0,018 & 0,000 & 0,022 \\
\hline$x+x 2+x 3$ & 1060100 & 0,018 & 0,012 & 0,000 & 0,016 \\
\hline$x+x 2+x 3$ & 1090100 & 0,034 & 0,022 & 0,000 & 0,027 \\
\hline$x+x 2+x 3$ & 306090 & 0,025 & 0,016 & 0,000 & 0,020 \\
\hline$x+x 2+x 3$ & 3060100 & 0,016 & 0,011 & 0,000 & 0,014 \\
\hline$x+x 2+x 3$ & 3090100 & 0,024 & 0,015 & 0,000 & 0,018 \\
\hline$x+x 2+x 3$ & 6090100 & 0,054 & 0,032 & 0,000 & 0,039 \\
\hline
\end{tabular}

Table S.2 : Same as Table S.1 for ozone daily maximum for the month of 201706.

\begin{tabular}{lllllll} 
AI & AR & IR & TA & TI & TR & model \\
\hline 0,355 & 0,102 & 0,051 & 0,244 & 0,069 & 0,039 & 1 \\
0,355 & 0,102 & 0,051 & 0,244 & 0,069 & 0,039 & 2 \\
0,878 & 0,286 & 0,158 & 0,651 & 0,271 & 0,090 & 3 \\
1,025 & 0,289 & 0,147 & 0,704 & 0,283 & 0,091 & 4
\end{tabular}

Table S.3 : Relative error (\%) of the PM10 univariate polynomial surrogate models averaged over Western Europe for three air pollution episodes $(201503,201612,201701)$ when including or excluding interaction terms. A color shading is applied to highlight the worst (red) and best (green) performances 


\begin{tabular}{lllllll} 
AI & AR & IR & TA & TI & TR & model \\
\hline 0,011 & 0,001 & 0,003 & 0,008 & 0,032 & 0,005 & 1 \\
0,011 & 0,001 & 0,003 & 0,008 & 0,032 & 0,005 & 2 \\
0,017 & 0,001 & 0,012 & 0,022 & 0,317 & 0,019 & 3 \\
0,020 & 0,002 & 0,011 & 0,019 & 0,312 & 0,018 & 4
\end{tabular}

Table S.4 : Same as Table S.3 for ozone daily maximum for the month of 201706.

$\begin{array}{lllllll}\text { AI } & \text { AR } & \text { IR } & \text { TA } & \text { TI } & \text { TR } & \text { model } \\ 1,003 & 0,941 & 0,128 & 0,535 & 0,067 & 0,057 & 1 \\ 0,638 & 0,558 & 0,072 & 0,614 & 0,074 & 0,028 & 2 \\ 0,884 & 0,781 & 0,095 & 0,519 & 0,060 & 0,031 & 3 \\ 0,996 & 0,832 & 0,127 & 0,353 & 0,128 & 0,054 & 4\end{array}$

Table S.5 : Relative error (\%) of the PM10 univariate polynomial surrogate models averaged over Western Europe for three air pollution episodes $(201503,201612,201701)$ when using different interaction sensitivity scenarios. A color shading is applied to highlight the worst (red) and best (green) performances

$\begin{array}{lllllll}\text { AI } & \text { AR } & \text { IR } & \text { TA } & \text { TI } & \text { TR } & \text { Model } \\ 0,047 & 0,037 & 0,045 & 0,065 & 0,049 & 0,060 & 1 \\ 0,040 & 0,022 & 0,026 & 0,048 & 0,034 & 0,034 & 2 \\ 0,047 & 0,030 & 0,036 & 0,062 & 0,037 & 0,047 & 3 \\ 0,037 & 0,031 & 0,034 & 0,048 & 0,153 & 0,043 & 4\end{array}$

Table S.6 : Same as Table S.5 for O3max 


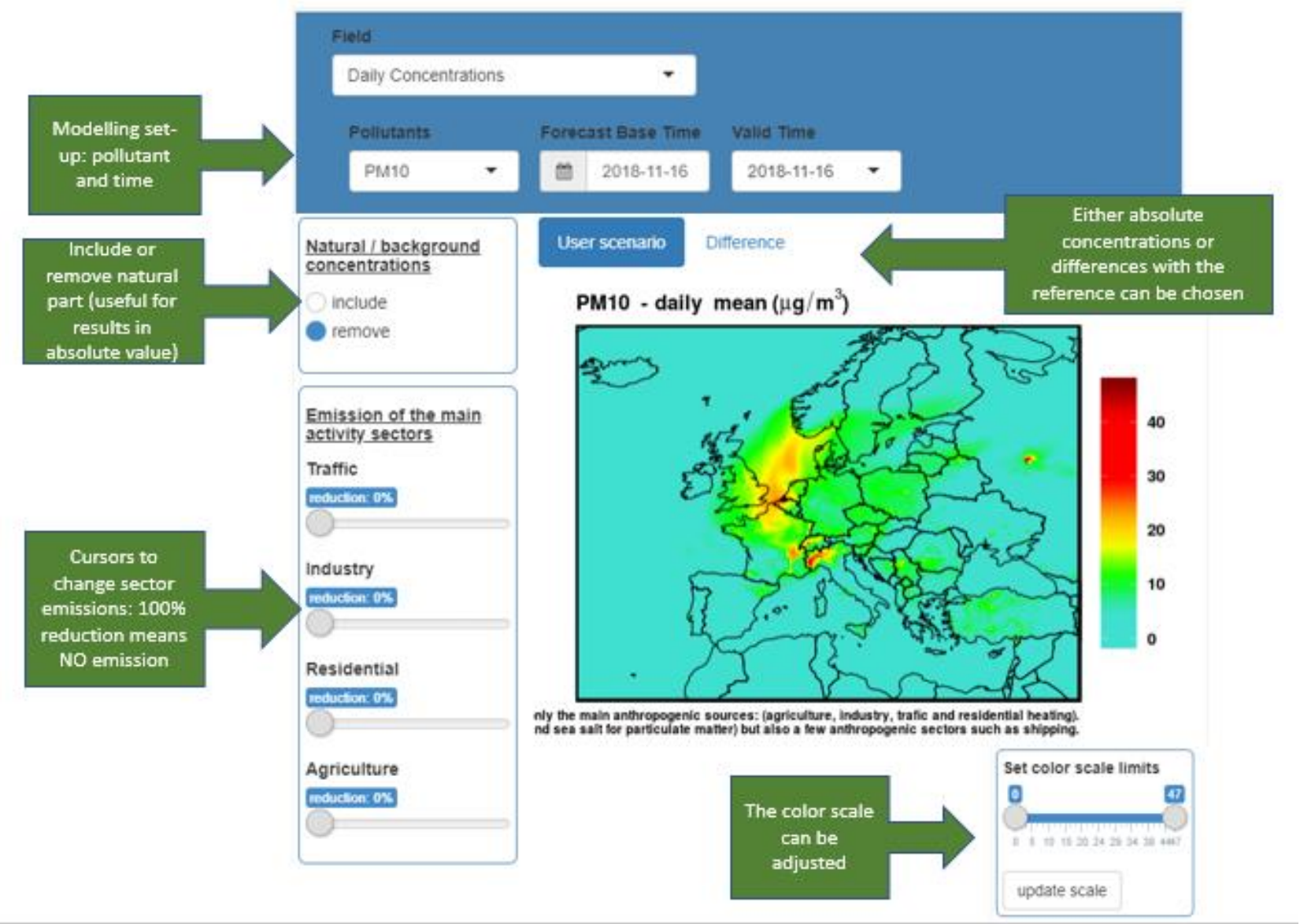

Figure S.9: Screenshot of the ACT web interface, https://policy.atmosphere.copernicus.eu/CAMS_ACT.php. 